\title{
Truncated fractional moments of stable laws
}

\author{
John P. Nolan ${ }^{1}$ \\ American University
}

\begin{abstract}
Expressions are given for the truncated fractional moments $E X_{+}^{p}$ of a general stable law. These involve families of special functions that arose out of the study of multivariate stable densities and probabilities. As a particular case, an expression is given for $E(X-a)_{+}$when $\alpha>1$.
\end{abstract}

Keywords: stable distribution, truncated moments, fractional moments 2000 MSC: 60E07, 60E10

\section{Introduction}

A univariate stable r.v. $Z$ with index $\alpha$, skewness $\beta$, scale $\gamma$, and location $\delta$ has characteristic function

$$
\phi(u)=\phi(u \mid \alpha, \beta, \gamma, \delta)=E \exp (i u Z)=\exp \left(-\gamma^{\alpha}\left[|u|^{\alpha}+i \beta \eta(u, \alpha)\right]+i u \delta\right),
$$

where $0<\alpha \leq 2,-1 \leq \beta \leq 1, \gamma>0, \delta \in \mathbb{R}$ and

$$
\eta(u, \alpha)= \begin{cases}-(\operatorname{sign} u) \tan (\pi \alpha / 2)|u|^{\alpha} & \alpha \neq 1 \\ (2 / \pi) u \ln |u| & \alpha=1 .\end{cases}
$$

In the notation of Samorodnitsky and Taqqu (1994), this is a $S_{\alpha}(\gamma, \beta, \delta)$ distribution. We will use the notation $X \sim \mathbf{S}(\alpha, \beta, \gamma, \delta ; 1)$ (the ";1" is used to distinguish between this parameterization and a continuous one used below).

The purpose of this paper is to derive expressions for truncated fractional moments $E X_{+}^{p}=E\left(X \mathbb{1}_{\{X \geq 0\}}\right)^{p}$ for general stable laws. To do this, define the functions for real $x$ and $d$

$g_{d}(x \mid \alpha, \beta)= \begin{cases}\int_{0}^{\infty} \cos (x r+\beta \eta(r, \alpha)) r^{d-1} e^{-r^{\alpha}} d r & 0<d<\infty \\ \int_{0}^{\infty}[\cos (x r+\beta \eta(r, \alpha))-1] r^{d-1} e^{-r^{\alpha}} d r & -2 \min (1, \alpha)<d \leq 0\end{cases}$

\footnotetext{
Email address: jpnolan@american.edu (John P. Nolan)

${ }^{1}$ The author was supported by an agreement with Cornell University, Operations Research \& Information Engineering under W911NF-12-1-0385 from the Army Research Development and Engineering Command.
} 


$$
\widetilde{g}_{d}(x \mid \alpha, \beta)= \begin{cases}\int_{0}^{\infty} \sin (x r+\beta \eta(r, \alpha)) r^{d-1} e^{-r^{\alpha}} d r & -\min (1, \alpha)<d<\infty \\ \int_{0}^{\infty}[\sin (x r+\beta \eta(r, \alpha))-x r] r^{d-1} e^{-r^{\alpha}} d r & \alpha>1,-\alpha<d \leq-1 .\end{cases}
$$

The functions $g_{d}(\cdot \mid \alpha, \beta)$ and $\widetilde{g}_{d}(\cdot \mid \alpha, \beta)$, for integer subscripts $d=1,2,3, \ldots$ were introduced in Abdul-Hamid and Nolan (1998). (The notation was slightly different there: a factor of $(2 \pi)^{-d}$ was included in the definition and $g_{\alpha, d}(x, \beta)$ was used instead of $g_{d}(x \mid \alpha, \beta)$, while $q_{\alpha, 1}(x, \beta)$ was used instead of $\widetilde{g}_{1}(x \mid \alpha, \beta)$.)

The expressions for $E X_{+}^{p}$ will involve the functions $g_{-p}(\cdot \mid \alpha, \beta)$ and $\widetilde{g}_{-p}(\cdot \mid \alpha, \beta)$, i.e. negative values fractional values of the subscript $d$. Before proving that result, we show that the functions $g_{d}(\cdot \mid \alpha, \beta)$ and $\widetilde{g}_{d}(\cdot \mid \alpha, \beta)$ have multiple uses. For a standardized univariate stable law, Fourier inversion of the characteristic function shows that the d.f. and density are given by

$$
\begin{aligned}
F(x \mid \alpha, \beta)-F(0 \mid \alpha, \beta) & =\frac{1}{\pi}\left(\widetilde{g}_{0}(x \mid \alpha, \beta)-\widetilde{g}_{0}(0 \mid \alpha, \beta)\right) \\
f(x \mid \alpha, \beta) & =\frac{1}{\pi} g_{1}(x \mid \alpha, \beta) .
\end{aligned}
$$

We note that there are explicit formulas for $F(0 \mid \alpha, \beta)$ when $\alpha \neq 1$.

The $g_{d}(\cdot \mid \alpha, \beta)$ functions are used in a similar way to give $d$-dimensional stable densities, see Theorem 1 of Abdul-Hamid and Nolan (1998) (note that there is a sign mistake in that formula when $\alpha=1$ ), and Nolan (2017) uses both $g_{d}(\cdot \mid \alpha, \beta)$ and $\widetilde{g}_{d}(\cdot \mid \alpha, \beta)$ to give an expression for multivariate stable probabilities.

Another use of these functions is in conditional expectation of $X_{2}$ given $X_{1}=x$ when $\left(X_{1}, X_{2}\right)$ are jointly stable with zero shift and spectral measure $\Lambda$. In general, the conditional expectation is a complicated non-linear function; here it is restated in terms of these functions. If $\alpha>1$ or $(\alpha \leq 1$ and (5.2.4) in Samorodnitsky and Taqqu (1994) holds), then Theorems 5.2.2 and 5.2.3 in Samorodnitsky and Taqqu (1994) show that the conditional expectation exists for $x$ in the support of $X_{1}$ and is given by

$$
\begin{aligned}
& E\left(X_{2} \mid X_{1}=x\right)= \\
& \begin{cases}c_{1} x+c_{2}\left[\frac{1-\left(x / \gamma_{1}\right) \widetilde{g}_{1}\left(x / \gamma_{1} \mid \alpha, \beta_{1}\right)}{g_{1}\left(x / \gamma_{1} \mid \alpha, \beta_{1}\right) / \gamma_{1}}\right] & \alpha \neq 1 \\
c_{0}+c_{1} \frac{x-\mu_{1}}{\gamma_{1}}+c_{2} \frac{\widetilde{g}_{1}\left(\left(x-\mu_{1}\right) / \gamma_{1}-\left(2 \beta_{1} / \pi\right) \ln \gamma_{1} \mid 1, \beta_{1}\right)}{g_{1}\left(x / \gamma_{1} \mid 1, \beta_{1}\right)} & \alpha=1, \beta_{1} \neq 0 \\
c_{0}+c_{1} \frac{x-\mu_{1}}{\gamma_{1}} & \\
+c_{2}\left[\frac{\left(1-\ln \gamma_{1}\right) g_{1}\left(\left(x-\mu_{1}\right) / \gamma_{1} \mid 1,0\right)+h_{1}\left(\left(x-\mu_{1}\right) / \gamma_{1} \mid 1,0\right)}{g_{1}\left(x / \gamma_{1} \mid 1,0\right)}\right] & \alpha=1, \beta_{1}=0,\end{cases}
\end{aligned}
$$

where $\beta_{1}$ and $\gamma_{1}$ are the skewness and scale parameters of $X_{1}$, and the constants and function $h_{1}(\cdot \mid 1,0)$ are given by

$$
c_{0}=-\frac{2}{\pi} \int_{\mathbb{S}} s_{2} \ln \left|s_{1}\right| \Lambda(d \mathbf{s})
$$




$$
\begin{aligned}
& c_{1}= \begin{cases}\frac{\kappa_{1}+\beta_{1} \tan ^{2}(\pi \alpha / 2) \kappa_{2}}{\gamma_{1}^{\alpha}\left(1+\beta_{1}^{2} \tan ^{2}(\pi \alpha / 2)\right)} & \alpha \neq 1 \\
\kappa_{2} / \beta_{1} & \alpha=1, \beta_{1} \neq 0 \\
\kappa_{1} & \alpha=1, \beta_{1}=0\end{cases} \\
& c_{2}= \begin{cases}\frac{\tan (\pi \alpha / 2)\left(\kappa_{2}-\beta_{1} \kappa_{1}\right)}{\gamma_{1}^{\alpha}\left(1+\beta_{1}^{2} \tan ^{2}(\pi \alpha / 2)\right)} & \alpha \neq 1 \\
\left(\kappa_{2}-\beta_{1} \kappa_{1} / \beta_{1}\right. & \alpha=1, \beta_{1} \neq 0 \\
-2 \kappa_{2} / \pi & \alpha=1, \beta_{1}=0\end{cases} \\
& \kappa_{1}=\left[X_{2}, X_{1}\right]_{\alpha}= \begin{cases}\int_{\mathbb{S}} s_{2} s_{1}^{<\alpha-1>} \Lambda(d \mathbf{s}) \\
\int_{\mathbb{S}} s_{2} s_{1}^{<0>} \Lambda(d \mathbf{s})=\int_{\mathbb{S}} s_{2} \operatorname{sign}\left(s_{1}\right) \Lambda(d \mathbf{s}) & \alpha=1\end{cases} \\
& \kappa_{2}=\int_{\mathbb{S}} s_{2}\left|s_{1}\right|^{\alpha-1} \Lambda(d \mathbf{s}) \\
& \mu_{1}=-\frac{2}{\pi} \int_{\mathbb{S}} s_{1} \ln \left|s_{1}\right| \Lambda(d \mathbf{s}) \\
& h(x \mid 1,0)=\int_{0}^{\infty} \cos (x r)(\log r) e^{-r} d r .
\end{aligned}
$$

In the terms above, $\mathbb{S}$ is the unit circle and $\left[X_{2}, X_{1}\right]_{\alpha}$ is the $\alpha$-covariation. Note that if $\Lambda$ is symmetric, then $c_{0}=\kappa_{2}=\beta_{1}=\mu_{1}=0$, so $c_{2}=0$ and

$$
E\left(X_{2} \mid X_{1}=x\right)=\frac{\left[X_{2}, X_{1}\right]_{\alpha}}{\gamma_{1}^{\alpha}} x
$$

is linear.

\section{Truncated moments $E X_{+}^{p}$}

The main result of this paper is the following expression for the fractional truncated moment of a stable r.v.

Theorem 1. Let $X \sim \mathbf{S}(\alpha, \beta, \gamma, \delta ; 1)$ with any $0<\alpha<2$ and any $-1 \leq \beta \leq 1$ and set

$$
\delta^{*}= \begin{cases}\delta / \gamma & \alpha \neq 1 \\ \delta / \gamma+(2 / \pi) \beta \log \gamma & \alpha=1 .\end{cases}
$$

For $p<\alpha$, define $m^{p}(\alpha, \beta, \gamma, \delta)=E X_{+}^{p}$.

(a) When $p=0$,

$$
m^{0}(\alpha, \beta, \gamma, \delta)=P(X>0)=\frac{1}{2}-\frac{1}{\pi} \widetilde{g}_{0}\left(-\delta^{*} \mid \alpha, \beta\right) .
$$

When $0<p<\min (1, \alpha)$,

$$
\begin{array}{r}
m^{p}(\alpha, \beta, \gamma, \delta)=\gamma^{p} \frac{\Gamma(p+1)}{\pi}\left[\sin \left(\frac{\pi p}{2}\right)\left(\frac{\Gamma(1-p / \alpha)}{p}-g_{-p}\left(-\delta^{*} \mid \alpha, \beta\right)\right)\right. \\
\left.-\cos \left(\frac{\pi p}{2}\right) \widetilde{g}_{-p}\left(-\delta^{*} \mid \alpha, \beta\right)\right] .
\end{array}
$$


When $p=1<\alpha<2$,

$$
m^{p}(\alpha, \beta, \gamma, \delta)=\gamma\left[\frac{\delta^{*}}{2}+\frac{1}{\pi}\left(\Gamma(1-1 / \alpha)-g_{-1}\left(-\delta^{*} \mid \alpha, \beta\right)\right)\right] .
$$

When $1<p<\alpha<2$,

$$
\begin{aligned}
m^{p}(\alpha, \beta, \gamma, \delta)=\gamma^{p} \frac{\Gamma(p+1)}{\pi}\left[\sin \left(\frac{\pi p}{2}\right)\right. & \left(\frac{\Gamma(1-p / \alpha)}{p}-g_{-p}\left(-\delta^{*} \mid \alpha, \beta\right)\right) \\
& \left.+\cos \left(\frac{\pi p}{2}\right)\left(\frac{\delta^{*}}{\alpha} \Gamma((1-p) / \alpha)-\widetilde{g}_{-p}\left(-\delta^{*} \mid \alpha, \beta\right)\right)\right] .
\end{aligned}
$$

(b) $E X_{-}^{p}=E(-X)_{+}^{p}=m^{p}(\alpha,-\beta, \gamma,-\delta)$.

Proof (a) To simplify calculations, first assume $\gamma=1$; the adjustment for $\gamma \neq 1$ is discussed below. When $p=0, E X_{+}^{0}=\int_{0}^{\infty} 1 f(x) d x=P(X>0)$, and (2) and $\widetilde{g}_{0}(x \mid \alpha, \beta) \rightarrow \pi / 2$ as $x \rightarrow \infty$ gives the value in terms of $\widetilde{g}_{0}(\cdot \mid \alpha, \beta)$.

When $0<p<\min (1, \alpha)$, Corollary 2 of Pinelis (2011) with $k=\ell=0$ shows

$$
E X_{+}^{p}=\frac{\Gamma(p+1)}{\pi} \int_{0}^{\infty} \Re \frac{\phi(u)-1}{(i u)^{p+1}} d u .
$$

First assume $\alpha \neq 1$ and set $\zeta=\zeta(\alpha, \beta)=-\beta \tan \frac{\pi \alpha}{2}$ and restricting to $u>0$,

$$
\begin{aligned}
\frac{\phi(u)-1}{(i u)^{p+1}} & =\left(\left[e^{-u^{\alpha}(1+i \zeta)+i \delta u}-1\right](-i) e^{-i(\pi / 2) p}\right) u^{-p-1} \\
& =\left(-i\left(e^{-u^{\alpha}}\left[\cos \left(\delta u-\zeta u^{\alpha}\right)+i \sin \left(\delta u-\zeta u^{\alpha}\right)\right]-1\right) e^{-i(\pi / 2) p}\right) u^{-p-1} \\
& =\left(\left[e^{-u^{\alpha}} \sin \left(\delta u-\zeta u^{\alpha}\right)-i\left(e^{-u^{\alpha}} \cos \left(\delta u-\zeta u^{\alpha}\right)-1\right)\right]\left[\cos \left(\frac{\pi p}{2}\right)-i \sin \left(\frac{\pi p}{2}\right)\right]\right) u^{-p-1}
\end{aligned}
$$

And therefore

$$
\begin{aligned}
\Re \frac{\phi(u)-1}{(i u)^{p+1}=} & {\left[\cos \left(\frac{\pi p}{2}\right) e^{-u^{\alpha}} \sin \left(\delta u-\zeta u^{\alpha}\right)-\sin \left(\frac{\pi p}{2}\right)\left(e^{-u^{\alpha}} \cos \left(\delta u-\zeta u^{\alpha}\right)-1\right)\right] u^{-p-1} } \\
= & \cos \left(\frac{\pi p}{2}\right) \sin \left(\delta u-\zeta u^{\alpha}\right) u^{-p-1} e^{-u^{\alpha}} \\
& \quad-\sin \left(\frac{\pi p}{2}\right)\left(\left[\cos \left(\delta u-\zeta u^{\alpha}\right)-1\right] u^{-p-1} e^{-u^{\alpha}}+\left(e^{-u^{\alpha}}-1\right) u^{-p-1}\right)
\end{aligned}
$$

Integrating this from 0 to $\infty$, substituting $t=u^{\alpha}$ in the last term to get

$$
E X_{+}^{p}=\frac{\Gamma(p+1)}{\pi}\left[-\cos \left(\frac{\pi p}{2}\right) \widetilde{g}_{-p}(-\delta \mid \alpha, \beta)-\sin \left(\frac{\pi p}{2}\right)\left\{g_{-p}(-\delta \mid \alpha, \beta)-\Gamma(1-p / \alpha) / p\right\}\right] .
$$

Next consider $0<p<\alpha=1$. Use (3) again, so we need to simplify

$$
\frac{\phi(u)-1}{(i u)^{p+1}}=\left(\left[e^{-u(1+i \beta \eta(u, 1))+i \delta u}-1\right](-i) e^{-i(\pi / 2) p}\right) u^{-p-1}
$$




$$
\begin{aligned}
&=\left(-i\left(e^{-u}[\cos (\delta u-\beta \eta(u, 1))+i \sin (\delta u-\beta \eta(u, 1))]-1\right) e^{-i(\pi / 2) p}\right) u^{-p-1} \\
&=\left(\left[e^{-u} \sin (\delta u-\beta \eta(u, 1))-i\left(e^{-u} \cos (\delta u-\beta \eta(u, 1))-1\right)\right]\right. \\
&\left.\quad \times\left[\cos \left(\frac{\pi p}{2}\right)-i \sin \left(\frac{\pi p}{2}\right)\right]\right) u^{-p-1} \\
& \Re \frac{\phi(u)-1}{(i u)^{p+1}}= {\left[\cos \left(\frac{\pi p}{2}\right) e^{-u} \sin (\delta u-\beta \eta(u, 1))\right.} \\
&\left.\quad-\sin \left(\frac{\pi p}{2}\right)\left(e^{-u}(\cos (\delta u-\beta \eta(u, 1))-1)+\left(e^{u}-1\right)\right)\right] u^{-p-1} .
\end{aligned}
$$

Integrating from 0 to $\infty$ yields

$$
E X_{+}^{p}=\frac{\Gamma(p+1)}{\pi}\left[-\cos \left(\frac{\pi p}{2}\right) \widetilde{g}_{-p}(-\delta \mid 1, \beta)-\sin \left(\frac{\pi p}{2}\right)\left\{g_{-p}(-\delta \mid 1, \beta)-\Gamma(1-p) / p\right\}\right] .
$$

When $p=1<\alpha<2, E X$ exists and is equal to $\delta$. Using Corollary 2 of Pinelis (2011) with $k=1, \ell=0$ shows

$$
E X_{+}=\frac{1}{2} E X+\frac{\Gamma(2)}{\pi} \int_{0}^{\infty} \Re \frac{\phi(u)-1}{(i u)^{p+1}} d u=\frac{\delta}{2}+\frac{1}{\pi} \int_{0}^{\infty} \Re \frac{\phi(u)-1}{(i u)^{p+1}} d u .
$$

The integrand is the same as above, with $\cos \left(\frac{\pi p}{2}\right)=0$ and $\sin \left(\frac{\pi p}{2}\right)=1$, so

$$
E X_{+}=\frac{\delta}{2}-\frac{1}{\pi}\left[g_{-1}(-\delta \mid \alpha, \beta)-\Gamma(1-1 / \alpha)\right] .
$$

When $1<p<\alpha<2$, Corollary 2 of Pinelis (2011) with $k=\ell=1$ shows

$$
E X_{+}^{p}=\frac{\Gamma(p+1)}{\pi} \int_{0}^{\infty} \Re \frac{\phi(u)-1-i u E X}{(i u)^{p+1}} d u
$$

Since $\alpha>1, E X$ exists and is equal to $\delta$. As above, for $u>0$,

$$
\begin{aligned}
\frac{\phi(u)-1-i u \delta}{(i u)^{p+1}=} & \left(\left[e^{-u^{\alpha}\left(1+i \zeta u^{\alpha}\right)+i \delta u}-1-i \delta u\right](-i) e^{-i(\pi / 2) p}\right) u^{-p-1} \\
= & \left(-i\left(e^{-u^{\alpha}}\left[\cos \left(\delta u-\zeta u^{\alpha}\right)+i \sin \left(\delta u-\zeta u^{\alpha}\right)\right]-1-i \delta u\right) e^{-i(\pi / 2) p}\right) u^{-p-1} \\
= & {\left[\left(e^{-u^{\alpha}} \sin \left(\delta u-\zeta u^{\alpha}\right)-\delta u\right)-i\left(e^{-u^{\alpha}} \cos \left(\delta u-\zeta u^{\alpha}\right)-1\right)\right] } \\
& \quad \times\left[\cos \left(\frac{\pi p}{2}\right)-i \sin \left(\frac{\pi p}{2}\right)\right] u^{-p-1}
\end{aligned}
$$

And therefore

$$
\begin{aligned}
\Re \frac{\phi(u)-1-i u \delta}{(i u)^{p+1}=}[ & \cos \left(\frac{\pi p}{2}\right)\left(e^{-u^{\alpha}} \sin \left(\delta u-\zeta u^{\alpha}\right)-\delta u\right) \\
& \left.\quad-\sin \left(\frac{\pi p}{2}\right)\left(e^{-u^{\alpha}} \cos \left(\delta u-\zeta u^{\alpha}\right)-1\right)\right] u^{-p-1} \\
= & \cos \left(\frac{\pi p}{2}\right)\left(\left[\sin \left(\delta u-\zeta u^{\alpha}\right)-\delta u\right] u^{-p-1} e^{-u^{\alpha}}+\delta\left(e^{-u^{\alpha}}-1\right) u^{-p}\right) \\
& \quad-\sin \left(\frac{\pi p}{2}\right)\left(\left[\cos \left(\delta u-\zeta u^{\alpha}\right)-1\right] u^{-p-1} e^{-u^{\alpha}}+\left(e^{-u^{\alpha}}-1\right) u^{-p-1}\right)
\end{aligned}
$$


Plugging this into (4) and integrating yields

$$
\begin{aligned}
& E X_{+}^{p}=\frac{\Gamma(p+1)}{\pi}\left\{\cos \left(\frac{\pi p}{2}\right)\left[-\widetilde{g}_{-p}(-\delta \mid \alpha, \beta)\right)+(\delta / \alpha)(\Gamma((1-p) / \alpha)]\right. \\
& \left.+\sin \left(\frac{\pi p}{2}\right)\left[-g_{-p}(-\delta \mid \alpha, \beta)+\Gamma(1-p / \alpha) / p\right]\right\}
\end{aligned}
$$

Now consider $\gamma \neq 1$. If $X \sim \mathbf{S}(\alpha, \beta, \gamma, \delta ; 1)$, then $X \stackrel{d}{=} \gamma Y$, where $Y \sim$ $\mathbf{S}\left(\alpha, \beta, 1, \delta^{*} ; 1\right)$, so $E X_{+}^{p}=\gamma^{p} E Y_{+}^{p}$. In symbols,

$$
m^{p}(\alpha, \beta, \gamma, \delta)=\gamma^{p} m^{p}\left(\alpha, \beta, 1, \delta^{*}\right) .
$$

(b) This follows from $-X \sim \mathbf{S}(\alpha,-\beta, \gamma,-\delta ; 1)$.

When $-1<p<0$, we conjecture that

$$
m^{p}(\alpha, \beta, \gamma, \delta)=\gamma^{p} \frac{\Gamma(p+1)}{\pi}\left[-\sin \left(\frac{\pi p}{2}\right) g_{-p}\left(-\delta^{*} \mid \alpha, \beta\right)-\cos \left(\frac{\pi p}{2}\right) \widetilde{g}_{-p}\left(-\delta^{*} \mid \alpha, \beta\right)\right] .
$$

\section{Related results}

There are several corollaries to the preceding result. First, taking $p=1$ in the previous result shows the following.

Corollary 2. If $X \sim \mathbf{S}(\alpha, \beta, \gamma, \delta ; 1)$ with $\alpha>1,-1 \leq \beta \leq 1, a \in \mathbb{R}$

$$
E(X-a)_{+}=\frac{\delta-a}{2}+\frac{\gamma}{\pi}\left[\Gamma\left(1-\frac{1}{\alpha}\right)-g_{-1}\left(\frac{\delta-a}{\gamma} \mid \alpha, \beta\right)\right] .
$$

Combining parts (a) and (b) of Theorem 1 yields.

Corollary 3. If $X \sim \mathbf{S}(\alpha, \beta, \gamma, \delta ; 1)$ with $0<\alpha<2,-1 \leq \beta \leq 1,-1 \leq p<\alpha$.

$$
\begin{aligned}
E|X|^{p} & =\gamma^{p} \frac{2 \Gamma(p+1)}{\pi} \sin \left(\frac{\pi p}{2}\right) \\
E X^{<p>} & =\gamma^{p} \frac{2 \Gamma(p+1)}{\pi} \cos \left(\frac{\pi p}{2}\right) \\
& \left.\left(\frac{\delta^{*} \Gamma((1-p) / \alpha)}{\alpha} \mathbb{1}_{\{p>1}\right\}-\widetilde{g}_{-p}\left(-\delta^{*} \mid \alpha, \beta\right)\right) .
\end{aligned}
$$

Proof $E|X|^{p}=E X_{-}^{p}+E X_{+}^{p}=m^{p}(\alpha, \beta, \gamma, \delta)+m^{p}(\alpha,-\beta, \gamma,-\delta)$ and $E X^{<p>}=$ $m^{p}(\alpha, \beta, \gamma, \delta)-m^{p}(\alpha,-\beta, \gamma,-\delta)$. Use Theorem 1 and the reflection property: $g_{d}(-x \mid \alpha, \beta)=g_{d}(x \mid \alpha,-\beta)$. Note that as $p \rightarrow 0, E|X|^{p} \rightarrow E 1=1$ and $E X^{<p>} \rightarrow-(2 / \pi) \widetilde{g}_{0}\left(\delta^{*} \mid \alpha, \beta\right)=P(X>0)-P(X<0)=1-2 F(0)$. Also as $p \rightarrow 1, E X^{<p>} \rightarrow \delta$.

In the strictly stable case, the expressions for $E X_{+}^{p}$ can be simplified using closed form expressions for $g_{d}(0 \mid \alpha, \beta)$ and $\widetilde{g}_{d}(0 \mid \alpha, \beta)$ when $\alpha \neq 1$. To state the result, set

$$
\theta_{0}=\theta_{0}(\alpha, \beta)= \begin{cases}\alpha^{-1} \arctan \left(\beta \tan \frac{\pi \alpha}{2}\right) & \alpha \neq 1 \\ \pi / 2 & \alpha=1 .\end{cases}
$$


Lemma 4. When $\alpha \neq 1$,

$$
\begin{aligned}
& g_{d}(0 \mid \alpha, \beta)= \begin{cases}\left(\cos \alpha \theta_{0}\right)^{d / \alpha} \cos \left(d \theta_{0}\right) \Gamma(1+d / \alpha) / d & d>0 \\
\left(\ln \left(\cos \alpha \theta_{0}\right)\right) / \alpha & d=0 \\
{\left[\left(\cos \alpha \theta_{0}\right)^{d / \alpha} \cos \left(d \theta_{0}\right)-1\right] \Gamma(1+d / \alpha) / d} & -\alpha<d<0\end{cases} \\
& \tilde{g}_{d}(0 \mid \alpha, \beta)= \begin{cases}-\left(\cos \alpha \theta_{0}\right)^{d / \alpha} \sin \left(d \theta_{0}\right) \Gamma(1+d / \alpha) / d & d \in(-\alpha, 0) \cup(0, \infty) \\
-\theta_{0} & d=0 .\end{cases}
\end{aligned}
$$

Proof Substitute $u=r^{\alpha}$ in the expressions for $g_{d}(0 \mid \alpha, \beta)$ and $\widetilde{g}_{d}(0 \mid \alpha, \beta)$. Then use respectively the integrals 3.944.6, 3.948.2, 3.945.1, 3.944.5, and 3.948.1 pg. 492-493 of Gradshteyn and Ryzhik (2000). (Note that some of these formulas have mistyped exponents.) Finally, when $\alpha \neq 1, \alpha \theta_{0}=-\arctan \zeta$, and for the allowable values of $\alpha$ and $\theta_{0}$,

$$
\cos \alpha \theta_{0}=\left|\cos \alpha \theta_{0}\right|=\left(1+\tan ^{2} \alpha \theta_{0}\right)^{-1 / 2}=\left(1+\zeta^{2}\right)^{-1 / 2} .
$$

The following is a different proof of Theorem 2.6.3 of Zolotarev (1986).

Corollary 5. Let $X$ be strictly stable, e.g. $X \sim \mathbf{S}(\alpha, \beta, \gamma, 0 ; 1)$ with $\alpha \neq 1$ or $(\alpha=1$ and $\beta=0)$ and $0<p<\alpha$.

(a) The fractional moment of the positive part of $X$ is

$$
E X_{+}^{p}=\frac{\gamma^{p}}{\left(\cos \alpha \theta_{0}\right)^{p / \alpha}} \frac{\Gamma(1-p / \alpha)}{\Gamma(1-p)} \frac{\sin p\left(\pi / 2+\theta_{0}\right)}{\sin (p \pi)} .
$$

(b) The fractional moment of the negative part of $X$ is $E X_{-}^{p}=E(-X)_{+}^{p}$, which can be obtained from the right hand side above by replacing $\theta_{0}$ with $-\theta_{0}$.

When $p=1$, the product $\Gamma(1-p) \sin (\pi p)$ in the denominator above is interpreted as the limiting value as $p \rightarrow 1$, which is $\pi$.

Proof Note that when $X$ is strictly stable, $\delta^{*}=0$. First assume $0<p<$ $\min (1, \alpha)$ and substitute Lemma 4 into this case of Theorem 1

$$
\begin{aligned}
E X_{+}^{p}= & \frac{\gamma^{p} \Gamma(p+1)}{\pi}\left[\sin (\pi p / 2)\left(\frac{\Gamma(1-p / \alpha)}{p}-\left(\left(\cos \alpha \theta_{0}\right)^{-p / \alpha} \cos \left(-p \theta_{0}\right)-1\right) \frac{\Gamma(1-p / \alpha)}{-p}\right)\right. \\
& \quad-\cos (\pi p / 2)\left(-\cos \left(\alpha \theta_{0}\right)^{-p / \alpha} \sin \left(-p \theta_{0}\right) \frac{\Gamma(1-p / \alpha)}{-p}\right] \\
= & \frac{\gamma^{p} \Gamma(p+1) \Gamma(1-p / \alpha)}{\pi p\left(\cos \alpha \theta_{0}\right)^{p / \alpha}}\left[\sin (\pi p / 2) \cos \left(p \theta_{0}\right)+\cos (\pi p / 2) \sin \left(p \theta_{0}\right]\right. \\
= & \frac{\gamma^{p} \Gamma(p+1) \Gamma(1-p / \alpha)}{\pi p\left(\cos \alpha \theta_{0}\right)^{p / \alpha}} \sin \left(\pi p / 2+p \theta_{0}\right)
\end{aligned}
$$


Using the identity $\Gamma(p+1)=\pi p /(\Gamma(1-p) \sin p \pi)$ gives the result.

When $p=1<\alpha$, again using the appropriate part of Theorem 1 shows

$$
\begin{aligned}
E X_{+} & =\gamma\left[0+\frac{1}{\pi}\left(\Gamma(1-1 / \alpha)-\left(\left(\cos \alpha \theta_{0}\right)^{-1 / \alpha} \cos \left(-\theta_{0}\right)-1\right) \frac{\Gamma(1-1 / \alpha)}{-1}\right)\right] \\
& \left.=\frac{\gamma \Gamma(1-1 / \alpha)}{\pi}\left[\cos \alpha \theta_{0}\right)^{-1 / \alpha} \cos \left(\theta_{0}\right)\right] .
\end{aligned}
$$

When $1<p<\alpha$, using Theorem 1 and $\delta^{*}=0$,

$$
\begin{aligned}
E X_{+}^{p}=\frac{\gamma^{p} \Gamma(p+1)}{\pi}[ & \sin (\pi p / 2)\left(\frac{\Gamma(1-p / \alpha)}{p}-\left(\left(\cos \alpha \theta_{0}\right)^{-p / \alpha} \cos \left(-p \theta_{0}\right)-1\right) \frac{\Gamma(1-p / \alpha)}{-p}\right) \\
& \left.+\cos (\pi p / 2)\left(0-\cos \left(\alpha \theta_{0}\right)^{-p / \alpha} \sin \left(-p \theta_{0}\right)\right) \frac{\Gamma(1-p / \alpha)}{-p}\right],
\end{aligned}
$$

and the rest is like the first case.

The standard parameterization used above is discontinuous in the parameters near $\alpha=1$, and it is not a scale-location family when $\alpha=1$. To avoid this, a continuous parameterization that is a scale-location family can be used. We will say $X \sim \mathbf{S}(\alpha, \beta, \gamma, \delta ; 0)$ if it has characteristic function

$E \exp (i u X)= \begin{cases}\exp \left(-\gamma^{\alpha}|u|^{\alpha}\left[1+i \beta\left(\tan \frac{\pi \alpha}{2}\right)(\operatorname{sign} u)\left(|\gamma u|^{1-\alpha}-1\right)\right]+i \delta u\right) & \alpha \neq 1 \\ \exp (-\gamma|u|[1+i \beta(2 / \pi)(\operatorname{sign} u) \log (\gamma|u|)]+i \delta u) & \alpha=1 .\end{cases}$

A stable r. v. $X$ can be expressed in both the 0-parameterization and the 1parameterization, in which case the index $\alpha$, the skewness $\beta$ and the scale $\gamma$ are the same. The only difference is in the location parameter: if $X$ is simultaneously $\mathbf{S}\left(\alpha, \beta, \gamma, \delta_{0} ; 0\right)$ and $\mathbf{S}\left(\alpha, \beta, \gamma, \delta_{1} ; 1\right)$, then the shift parameters are related by

$$
\delta_{1}= \begin{cases}\delta_{0}-\beta \gamma \tan \frac{\pi \alpha}{2} & \alpha \neq 1 \\ \delta_{0}-(2 / \pi) \beta \gamma \log \gamma & \alpha=1 .\end{cases}
$$

Therefore, if $X \sim \mathbf{S}\left(\alpha, \beta, \gamma, \delta_{0} ; 0\right)$,

$$
E X_{+}^{p}= \begin{cases}m^{p}\left(\alpha, \beta, \gamma, \delta_{0}-\beta \gamma \tan \frac{\pi \alpha}{2}\right) & \alpha \neq 1 \\ m^{p}\left(\alpha, \beta, \gamma, \delta_{0}-(2 / \pi) \beta \gamma \log \gamma\right) & \alpha=1\end{cases}
$$

This quantity is continuous in all parameters.

For the above expressions for $E X_{+}^{p}$ to be of practical use, one must be able to evaluate $g_{d}(\cdot \mid \alpha, \beta)$ and $\widetilde{g}_{d}(\cdot \mid \alpha, \beta)$. When $d$ is a nonnegative integer, Nolan (2017) gives Zolotarev type integral expressions for these functions. However, this is not helpful here, where negative, non-integer values of $d$ are needed. We have written a short $\mathrm{R}$ program to numerically evaluate the defining integrals for $g_{d}(\cdot \mid \alpha, \beta)$ and $\widetilde{g}_{d}(\cdot \mid \alpha, \beta)$. A single evaluation takes less than 0.0002 seconds on a modern desktop. This faster than numerically evaluating $E X_{+}^{p}=$ $\int_{0}^{\infty} x^{p} f(x \mid \alpha, \beta, \gamma, \delta) d x$, because the latter requires many numerical calculations of the density $f(x \mid \alpha, \beta, \gamma, \delta)$. 


\section{References}

\section{References}

Abdul-Hamid, H., Nolan, J. P., 1998. Multivariate stable densities as functions of one dimensional projections. J. Multivar. Anal. 67, 80-89.

Gradshteyn, I., Ryzhik, I., 2000. Table of Integrals, Series, and Products. Academic Press.

Nolan, J. P., 2017. Multivariate stable cumulative probabilities in polar form and related functions, in progress.

Pinelis, I., 2011. Positive-part moments via the Fourier-Laplace transform. Journal of Theoretical Probability 24 (2), 409-421. URL http://dx.doi.org/10.1007/s10959-010-0276-9

Samorodnitsky, G., Taqqu, M., 1994. Stable Non-Gaussian Random Processes. Chapman and Hall, New York.

Zolotarev, V. M., 1986. One-dimensional Stable Distributions. Vol. 65 of Translations of Mathematical Monographs. American Mathematical Society, Translation of the original 1983 Russian edition. 